\title{
A Case of Cold Urticaria Mistaken as Congestion Status during Breast Reconstruction with Deep Inferior Epigastric Perforator Free Flap Coverage
}

\section{Yoon Jae Lee, Hyun Jun Cho, Deuk Young Oh}

Department of Plastic and Reconstructive Surgery, Seoul St. Mary's Hospital, College of Medicine, The Catholic University of Korea, Seoul, Korea
No potential conflict of interest relevant to this article was reported.
We present the first reported case of cold urticaria, which was mistaken as congestion status, in a patient undergoing breast reconstruction with deep inferior epigastric perforator (DIEP) flap coverage. A 53-year-old woman underwent an immediate bilateral breast reconstruction with DIEP flap coverage. The harvested DIEP flap was wrapped with wet gauze and kept above an ice pack to minimize ischemic damage before flap in-setting to the mastectomy site. After the transfer of the harvested DIEP flap, she had suddenly developed an erythematous and edematous lesion, specifically on the flap. The room temperature was raised and a single dose of steroid and antihistamine was given intravenously. At the first instance, flap congestion was suspected; however, as the rash gradually spread throughout the body, we could deduce that it was cold urticaria. The erythematous rash and whirl resolved soon after receiving medication. Patients with known cold urticaria undergoing surgical procedures require several precautions (e.g., antihistamine, relatively warm operation room) to lessen the risk of severe reaction. Especially in patients with DIEP free flap, the lack of preoperative treatment could result in a pinkish flap due to urticaria, which could be mistaken by the surgeon as a congested flap.

Keywords Breast, Free tissue flaps, Hyperemia, Urticaria

\section{INTRODUCTION}

Although the exact pathogenesis of cold urticaria is unknown, its symptoms of wheals and hives, which are conditions found predominantly in young women, are induced by a cold environment [1-3]. Cold urticaria diagnosis is based on careful history taking and the ice cube test [1-3]. The pinkish rash eruption is accompanied by vasodilation, which is mediated by the release of cutaneous

Received: Jan 11, 2017 Revised: Jan 24, 2017 Accepted: Jan 24, 2017 Correspondence: Deuk Young Oh Department of Plastic and Reconstructive Surgery, Seoul St. Mary's Hospital, College of Medicine, The Catholic University of Korea, 222 Banpo-daero, Seocho-gu, Seoul 06591, Korea. E-mail: ohdeuk1234@hanmail.net

Copyright () 2017 The Korean Society for Aesthetic Plastic Surgery.

This is an Open Access article distributed under the terms of the Creative Commons Attribution Non-Commercial License (http://creativecommons.org/licenses/by-nc/4.0/) which permits unrestricted non-commercial use, distribution, and reproduction in any medium, provided the original work is properly cited. $\quad w w w . e-a a p s . o r g$ mast cells, such as histamine [1-3]. In a free flap operation, the harvested flap is usually exposed to cold conditions before anastomosis to reduce ischemic injury, which could induce wheals and hives that could be mistaken as signs of flap congestion. The intraoperative appearance of a congested flap is similar to the clinical presentation of cold urticaria, which may arise subtly in the form of rapid capillary refill and cutaneous discoloration.

Here, we report a case of cold urticaria mistaken as flap congestion status in a patient undergoing deep inferior epigastric perforator (DIEP) free flap surgery, and discuss its proper management.

\section{CASE}

A 53-year-old woman presented for immediate breast reconstruction after bilateral mastectomy. She had a history of bilateral lumpectomy 5 years ago; however, because of recurrence, the patient had bilateral mastectomy (Fig. 1). Thereafter, the patient underwent breast reconstruction with DIEP free flap coverage under general 


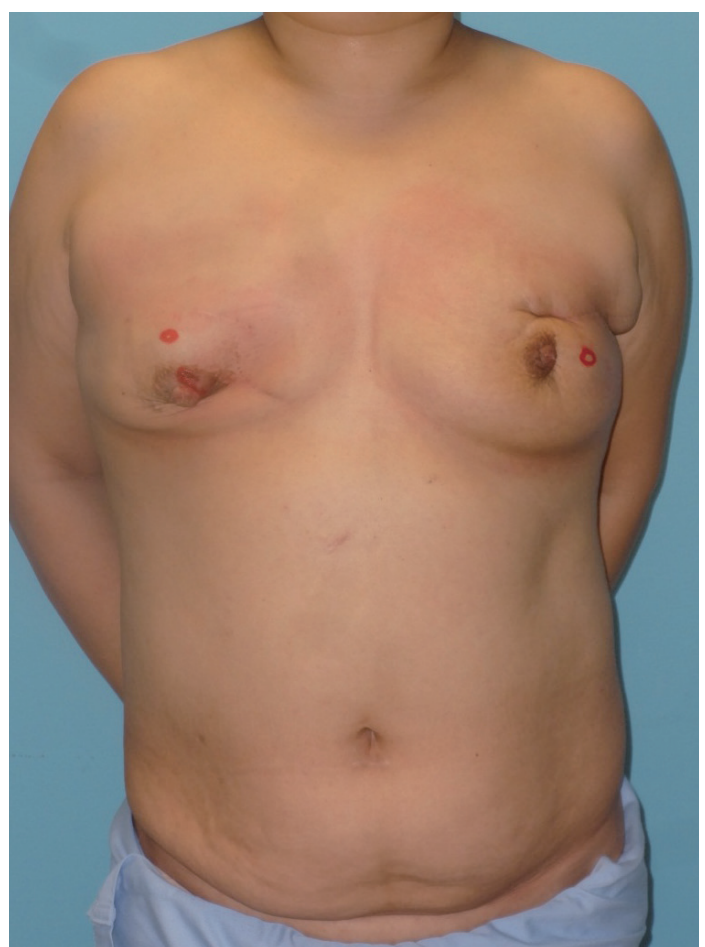

Fig. 1. Preoperative clinical photo. Asymmetry and dimpling as well as scarring of both breasts due to lumpectomy.

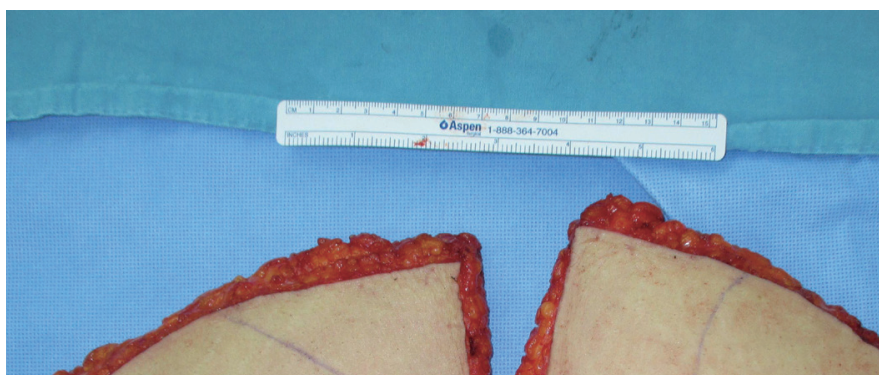

Fig. 2. Harvested deep inferior epigastric perforator flap, divided in half. Each contains a pedicle for bilateral flap coverage. Before wrapping the flap in wet gauze, its color was good and it has normal capillary refill.

anesthesia. The harvested DIEP flap was wrapped with wet gauze and kept above an ice pack before anastomosis to minimize ischemic damage before flap in-setting to the mastectomy site (Fig. 2). After completion of recipient vessel preparation, such as thoracodorsal artery and vein isolation, the ice-cooled harvested DIEP flap was transferred to the mastectomy site for anastomosis. Although the anastomosis was successful without any complication, the patient suddenly developed an erythematous and edematous lesion on the flap, which was initially attributed to congestion status (Fig. 3).

To confirm congestion status, a pin prick test was performed and fresh blood was noticed. Within few minutes, lesions similar to those seen on the flap spread to other areas, such as the abdo-

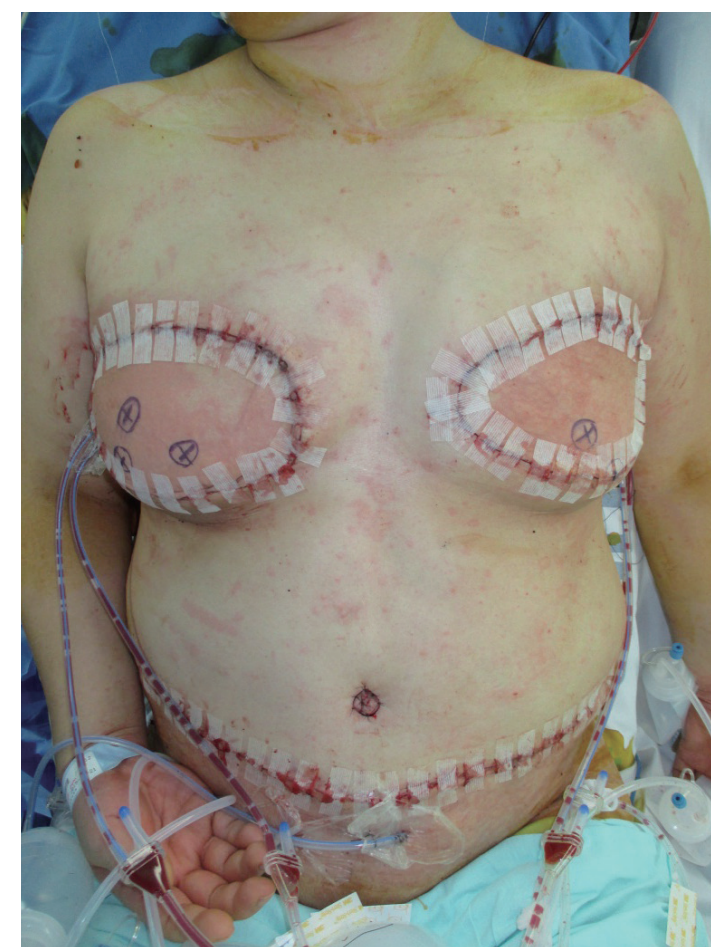

Fig. 3. Intraoperative clinical photo of the patient. Erythematous flap due to cold urticaria during operation, which was easy to be mistaken as due to congestion status.

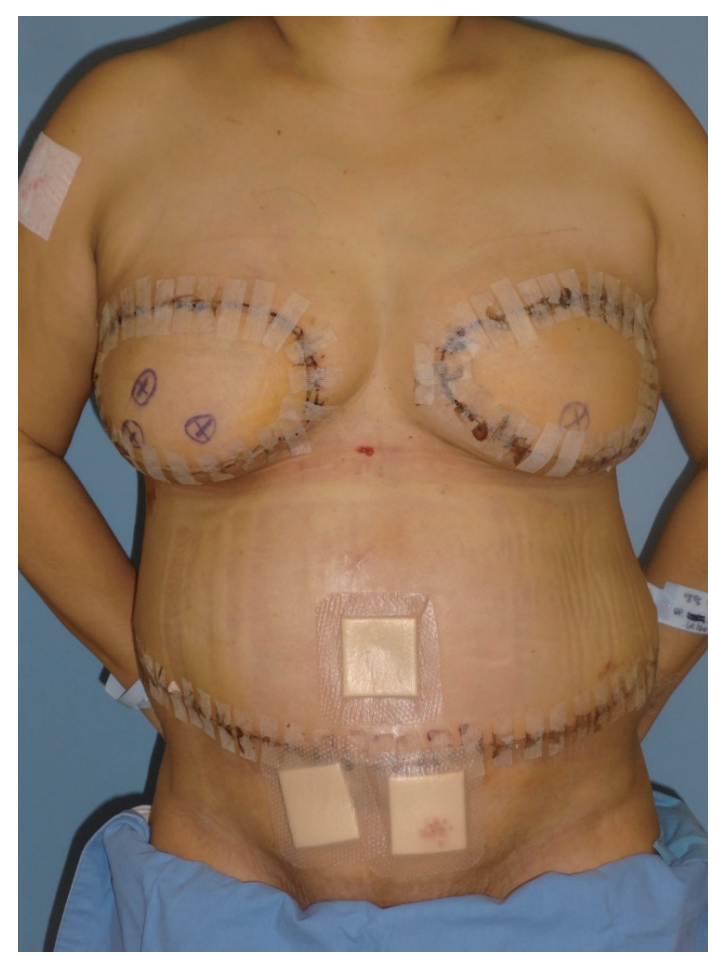

Fig. 4. Postoperative clinical photo of the patient after 7 days. The flap is stable with a good surgical outcome. 
men and both arms, and the lesions remained persistent throughout the operation. As the symptoms were typical of urticaria, the room temperature was raised and a single dose of steroid and antihistamine was administered intravenously. The patient had a good surgical recovery and was treated with diphenhydramine and prednisone (1 mg/kg/day) administered orally. Postoperative ice pack cooling was avoided. Eventually, the full resolution of the erythematous raised wheal was achieved over 24 hours after surgery. Therefore, the patient had cold urticaria that responded well to antihistamine therapy and ultimately had a good surgical outcome without long-term sequelae (Fig. 4).

\section{DISCUSSION}

Cold urticaria, whether familial or acquired, is a subtype of physical urticaria and characterized by the development of wheals and angioedema after cold exposure [1-3]. The mechanism involves mast cell degranulation and the subsequent release of histamine and proinflammatory cytokines upon exposure of the skin to a cold environment. Generally, these symptoms potentially have a benign course and are self-limited; however, in some conditions, they can be life-threatening, especially when systemic anaphylactic reaction and pharyngeal angioedema occur.

This study presents a case of transient cold urticaria that developed after keeping the harvested flap on ice-packed saline before anastomosis, which has not been reported previously. As the harvested flap immediately developed generalized swelling and erythema on the skin after anastomosis, congestion status was suspected. A pin prick test was performed and fresh blood was noticed, consequently leading to the true cause of the flap change, i.e., cold urticaria, which mimicked the signs of congestion, such as decreased capillary refill and discoloration.

The incidence of venous congestion status in DIEP and transverse rectus abdominis myocutaneous flaps for breast reconstruction is not rare, ranging from 2 to $20 \%$ [4-6]. The main causes of venous congestion are venous thrombosis, inadequate venous drainage, inadequate perforator selection, and anatomic superficial venous system dominance with insufficient communicating veins to the deep system [4-6]. In contrast, cold urticaria prevalence is not well determined and has an estimated incidence rate of $0.05 \%$ [3]. Although the incidence is low, identifying patients with cold urticaria is vital as anaphylactic shock may occur in some cases [7-9]. The clinical presentation of urticaria varies, ranging from localized raised wheal to severe systemic responses, such as hypotension, laryngeal edema, and anaphylactic shock [2]. Most cases are primary, and the secondary form includes cryoglobulinemia, cold hemolysin, malignancies, leukocytosis, and infectious diseases [1]. Therefore, excluding secondary causes before making a diagnosis of primary acquired cold urticaria is essential. Besides medical history taking, the cold stimulation test is compulsory to confirm cold ur- ticaria diagnosis. In this study, the patient was not intended for but tested positive to the ice cube test; the harvested flap covered by wet gauze was placed over the ice pack over 10 minutes, and after the ice pack was removed (the free flap was transferred to the anastomosis site), the exposed area developed a confluent wheal-andflare reaction. Normally, the ice pack is placed on the patient's forearm for 5 minutes; the skin is allowed to go back to its normal temperature for 10 minutes [1-3]. The test is positive when a wheal develops. In patients with known cold urticaria history, prophylactic antihistamine agents should be administered before the operation, and strict avoidance of ice cube cooling of the harvested flap is imperative. Furthermore, the operating room must be kept warm, and warmed intravenous fluids should be prepared. Surgeons should be aware of this cause of intraoperative swelling and erythematous change and should not mistake cold urticaria as congestion status, as symptoms induced by cold urticaria require an individually complex management.

\section{CONCLUSION}

We present the first case of cold urticaria after breast reconstruction with DIEP free flap coverage. There are no reports of urticaria or anaphylactic reactions occurring with DIEP free flap or other free flap surgery. Although ice cooling of the harvested flap may reduce ischemic injury, this should be avoided in patients with urticaria history; thus, early recognition is essential to minimize adverse effects, and cold urticaria should not be mistaken as congestion status.

\section{PATIENT CONSENT}

Patients provided written consent for the use of their images.

\section{REFERENCES}

1. Burroughs JR, Patrinely JR, Nugent JS, et al. Cold urticaria: an underrecognized cause of postsurgical periorbital swelling. Ophthal Plast Reconstr Surg 2005;21:327-30.

2. Booth K, Parissis H. Management of cold-induced urticaria during cardiac surgery. J Card Surg 2011;26:158-9.

3. Işk S, Arkan-Ayyldz Z, Sozmen SC, et al. Idiopathic cold urticaria and anaphylaxis. Pediatr Emerg Care 2014;30:38-9.

4. Sbitany H, Mirzabeigi MN, Kovach SJ, et al. Strategies for recognizing and managing intraoperative venous congestion in abdominally based autologous breast reconstruction. Plast Reconstr Surg 2012;129:80915.

5. Momeni A, Lee GK. A case of intraoperative venous congestion of the entire DIEP-flap--a novel salvage technique and review of the literature. Microsurgery 2010;30:443-6.

6. Galanis C, Nguyen P, Koh J, et al. Microvascular lifeboats: a stepwise 
approach to intraoperative venous congestion in DIEP flap breast reconstruction. Plast Reconstr Surg 2014;134:20-7.

7. Habib N, Saedi N, Zachary C. Cold-induced urticaria after fractional carbon dioxide laser resurfacing of the face. Dermatol Surg 2011;37: 1700-3.
8. Singleton R, Halverstam CP. Diagnosis and management of cold urticaria. Cutis 2016;97:59-62.

9. Greaves MW. Pathology and classification of urticaria. Immunol Allergy Clin North Am 2014;34:1-9. 